\title{
Les dieux d'Homère. Polythéisme et poésie en Grèce ancienne
}

(Kernos Supplément 31), Liège, Presses Universitaires de Liège, 2017, pp. 262, ISBN 978-2-87562-130-6, € 25,00

\section{Pierre Ellinger}

\section{OpenEdition}

\section{Journals}

Édition électronique

URL : http://journals.openedition.org/mythos/383

DOI : $10.4000 /$ mythos.383

ISSN : 2037-7746

Éditeur

Salvatore Sciascia Editore

\section{Édition imprimée}

Date de publication : 1 décembre 2018

Pagination : 166-168

ISBN : 978-88-8241-501-3

ISSN : 1972-2516

\section{Référence électronique}

Pierre Ellinger, «Les dieux d'Homère. Polythéisme et poésie en Grèce ancienne », Mythos [En ligne], 12 | 2018, mis en ligne le 24 septembre 2019, consulté le 23 septembre 2020. URL : http:// journals.openedition.org/mythos/383; DOI : https://doi.org/10.4000/mythos.383 


\section{Gabriella Pironti, Corinne Bonnet (éd.)}

Les dieux d'Homère. Polythéisme et poésie en Grèce ancienne

(Kernos Supplément 31), Liège, Presses Universitaires de Liège, 2017, pp. 262, ISBN 978-2-87562-130-6, € 25,00

Pierre Ellinger - Université Paris Diderot-USPC - pierre.ellinger@univ-paris-diderot.fr

Ce nouveau supplément de la revue Kernos est issu d'une rencontre internationale tenue à Rome en 2015. Le livre avait déjà fait l'objet d'une publication en italien aux éditions Carocci en 2016. Le voici en version française, qui aura probablement une plus large diffusion. Les textes en français sont redonnés cette fois dans l'original, les textes italiens traduits à leur tour. Comme l'indiquent les éditrices, Gabriella Pironti et Corinne Bonnet, dans leur introduction commune, le projet est de reprendre à nouveaux frais l'étude des dieux d'Homère, et cela au regard des avancées des recherches anthropologiques sur le polythéisme grec, loin des préjugés moralistes ou d'une visée purement littéraire où ils ne seraient qu'un banal ornement du récit que I'on pourrait éventuellement supprimer. Les poèmes et le monde que le poète essaie de penser à travers eux ne sauraient se comprendre sans les dieux, sans le jeu complexe de leurs interrelations avec les hommes en même temps qu'entre eux, et sans essayer de démêler l'imbrication étroite des actions des uns et des autres dans la narration. Pour ce faire, les neuf contributions réunies ici, regroupées en trois parties, abordent successivement les questions de la représentation des dieux chez Homère, de la communication des dieux entre eux et avec les hommes, et enfin des interactions des dieux et des hommes dans la guerre, particulièrement dans l'lliade.

Maurizio Bettini ouvre la première partie, "Raconter les puissances divines", en rappelant le privilège extraordinaire que le poète confère à son public de voir les dieux "en direct ", comme ils sont censés "être vraiment ", sous leur forme anthropomorphique. Les héros, au contraire, n'ont pas ordinairement un tel privilège. D'où le choix original, en un temps où les épiphanies sont devenues un sujet de recherche à la mode, de souligner plutôt les paradoxes de "l'apparaître " divin, sous forme le plus souvent d'invisibilité, "air ", brouillard, "nuage", nuit, obscurité. Cela ne veut pas dire, comme le remarque $M$. Bettini de manière plaisante, que de petits nuages se promènent parmi les combattants dans la plaine de Troie, mais que le champ de la vision humaine est affecté d'infirmité, de points aveugles, que les dieux ne consentent qu'exceptionnellement à réduire pour quelques protégés.

Après ce premier chapitre très neuf, le propos d'Adeline Grand-Clément, dans la suite de ses travaux sur les couleurs des Grecs, paraîtra plus classique. Elle rappelle que les dieux peuvent se distinguer les uns des autres aussi par leurs couleurs, mais elle attire surtout l'attention sur le côté polysensoriel de la perception des dieux par les humains, pas seulement leur éclat lumineux, mais tout à la fois le toucher, l'odeur, le son et même l'effet de souffle.

Finalement Gabriella Pironti revient au privilège homérique, et même à son summum dans I'lliade, lorsqu'Homère nous fait pénétrer jusque sous la couverture de nuage doré censée cacher aux autres dieux eux-mêmes les amours du souverain des dieux et de sa rusée épouse sur le mont Ida. Commentant l'épisode de la Dios apatè du chant XIV, où Héra parvient à endormir et tromper momentanément la vigilance de Zeus pour relancer la guerre, elle montre comment, à l'intérieur même du système relationnel du panthéon, la déesse réussit - du moins semble-t-il - à manipuler les pouvoirs fonctionnels de sa collègue et adversaire Aphrodite pour parvenir à ses fins. 
Sur la communication entre les hommes et les dieux, la deuxième partie, "Entre l'Olympe et la terre ", réunit à nouveau trois études. Corinne Bonnet compare de manière détaillée les assemblées humaines et divines chez Homère dans leurs ressemblances et différences. Ce chapitre, qui commence en évoquant la parodie d'assemblée divine chez Lucien (où Homère est originaire de Babylone!), se termine sur une autre comparaison très intéressante, qu'on aurait aimé voir plus développée, entre les assemblées divines homériques et celles proche-orientales en Mésopotamie, en Syrie à Ougarit, et même autour de Yaveh dans l'Ancien Testament.

Carmine Pisano traite, pour sa part, des envoyés occasionnels et messagers fonctionnels des dieux auprès des hommes. II compare principalement le mode d'action d'Iris et celui d'Hermès, lequel est beaucoup plus riche, non seulement dans son extension, pouvant se porter jusqu'aux extrémités du monde, mais surtout dans son autonomie et sa puissance de persuasion. Episodiquement, Iris peut se montrer aussi convaincante qu'Hermès, sans se contenter de répéter comme à son habitude mot pour mot la parole de Zeus : la "plasticité " du panthéon, concept un peu vague souvent employé dans ce livre, se réduit dans ce cas à une assez mince bande de recouvrement.

Les dieux d'Homère sont aussi des dieux du culte. Vinciane Pirenne-Delforge reprend donc l'analyse des scènes cultuelles de l'lliade, pour étudier la représentation qu'elles donnent de la communication rituelle des hommes en direction des dieux: la prière comme un pont jeté vers ceux-ci, et les scènes de sacrifice. Ces scènes homériques restent un témoignage essentiel pour appréhender le noyau central de la pratique rituelle grecque, pour autant qu'on ne néglige pas les impératifs de la narration et le contexte pour les comprendre, en particulier les variations par rapport au schéma liturgique de base énoncé dans les poèmes. Pour finir, V. Pirenne-Delforge commente l'offrande des Troyennes à Athéna dans son temple sur l'acropole de Troie. L'offrande du péplos posé sur les genoux de la statue de la déesse, la prière qui l'accompagne, correspondent, dit-elle, à la pratique de "n'importe quelle cité grecque historique ". Mais la réponse de la déesse à la requête, son refus (que les suppliantes n'entendent pas) répondraient à la seule logique narrative du poème qui veut la destruction de Troie. L'expérience des Troyennes a-t-elle été si unique, et non pas celle aussi de nombreuses cités grecques dans I'histoire, confrontées à la défaite et à l'éventualité de leur destruction? Dans la tension qui traverse la représentation du divin en contexte polythéiste entre perspective "olympienne " et particularismes des panthéons des cités, la vision panhellénique ne serait-elle pas plus réaliste que celle bornée des micro-panthéons locaux? Cléomène, le roi de Sparte - pour reprendre le dernier exemple invoqué dans ce chapitre -, qui a aussi son Athéna poliade, monte, en tant que descendant d'Agamemnon, à l'Acropole d'Athènes pour tester le soutien que pourrait lui apporter la déesse, et se voit répondre qu'il n'est pas du pays. Mais, un siècle plus tard, dans la nouvelle guerre panhellénique, au soir de la victoire de Lysandre à Aigos-Potamos, où se trouvait donc Athéna? A attendre déjà le retour de Thrasybule et la chute des Trente Tyrans?

On est donc introduit aux questions de la dernière partie, "De la guerre au salut ", qui portent, pour l'essentiel, comme on pouvait s'y attendre, sur les dieux d'Homère et la guerre. Pascal Payen souligne la complexité de la pensée de la guerre dans l'épopée, où l'éthique de la gloire fait simultanément l'objet d'une profonde et constante remise en cause. La figure d'Achille est ici centrale, qui, dans l'absolu de son renoncement au combat comme de ses excès guerriers, courbe autour d'elle, tout au long du poème, les lignes de l'action divine, dont on aurait pu supposer la maîtrise sans failles du conflit. La guerre était-elle si naturelle pour les Grecs ? C'est le débat lancé naguère par Arnaldo Momigliano que rouvre aussi David Bouvier, qui constate que si les monothéismes ont conduit aux guerres de religion, le polythéisme grec a pour sa part 
divinisé la guerre, ainsi que d'autres entités comme la vengeance, la querelle, les tueries (assurément, pour les Modernes, il serait moins "inquiétant" de diviniser seulement l'ordre ou la justice, Dikè ou Thémis). Mais si le polythéisme peut faire d'un nom commun une divinité, le moyen de comprendre un objet en devient-il aussi la cause ? Revenant de ces considérations un peu abstraites, D. Bouvier analyse le chant III de I'lliade, la tentative par le duel de Pâris et Ménélas pour arrêter la guerre. Le véritable vainqueur du duel est ici Aphrodite qui, en sauvant le Troyen vaincu et en forçant Hélène à s'unir à nouveau avec lui, rejoue et réitère sa victoire du jugement de Pâris, cause de la guerre qu'elle relance ainsi. La déesse de la philotès - le concept souligne à la fois la fragilité des relations humaines et les risques de la séduction - est pour les manipulations du roi des dieux, un agent fauteur de guerre beaucoup plus efficace qu'Athéna, ou l'irascible Héra et son digne fils Arès, le dieu de la folie meurtrière.

L'ouvrage s'achève par un dernier chapitre où Miguel Herrero de Jáuregui s'est proposé d'approfondir la question, à son avis trop négligée, de la puissance salvatrice des divinités homériques. II procède, pour ce faire, à partir des deux poèmes, à un commentaire très précis de la sentence prononcée par Athéna-Mentor à l'intention de Télémaque dans l'Odyssée : " Il est facile à un dieu, quand il le veut, de sauver un homme, et même de loin ". Le pouvoir salvateur des dieux d'Homère s'exerce dans le respect de la distinction mortalité/immortalité. Seuls les dieux, à la différence des hommes, peuvent "sauver", mais ils ne peuvent abolir la mort. Ils permettent à l'occasion, à leurs protégés, d'échapper à un danger ponctuel, en reconnaissance des services cultuels rendus, ou en fonction de la politique de l'Olympe, mais cela n'est jamais automatique, et trop de protection nuirait à la gloire des héros. Du point de vue des humains tout cela apparaît comme hasardeux - mais leur laisse sans doute à eux aussi leur marge d'action. Homère apparaît ainsi, encore une fois, comme un penseur plus réaliste, comparé, sinon à d'hypothétiques conceptions opposées du salut contemporaines, en tout cas à l'inflation ultérieure des titres sotériologiques des dieux.

Au total, même s'il n'est peut-être pas aussi inaugural que l'affirme l'introduction, on a ici un ensemble de travaux tout à fait intéressants, particulièrement quand ils se portent à la limite de nos questionnements sur le poIythéisme antique, et nous laissent entendre ce qui reste à explorer pour mieux comprendre le rôle qu'a pu jouer "Homère " pour les Grecs : leur offrir, comme le dit Pierre Judet de la Combe, dans son beau livre (Homère, Paris 2017, 235), paru la même année, la possibilité "d'expérimenter ce qu'ils ne connaîtront jamais, une expérience du tout de l'histoire humaine". 